\title{
Aspin-en-Lavedan, Lourdes, Ossen, Ségus
}

Prospection

Jean-Luc Boudartchouk

\section{(2) OpenEdition}

\section{Journals}

Édition électronique

URL : http://journals.openedition.org/adlfi/10819

ISSN : 2114-0502

Éditeur

Ministère de la culture

Référence électronique

Jean-Luc Boudartchouk, «Aspin-en-Lavedan, Lourdes, Ossen, Ségus », ADLFI. Archéologie de la FranceInformations [En ligne], Midi-Pyrénées, mis en ligne le 01 mars 1997, consulté le 19 avril 2019. URL http://journals.openedition.org/adlfi/10819

Ce document a été généré automatiquement le 19 avril 2019

(C) Ministère de la Culture et de la Communication, CNRS 


\title{
Aspin-en-Lavedan, Lourdes, Ossen, Ségus
}

Prospection

\author{
Jean-Luc Boudartchouk
}

Date de l'opération : 1991 (PR)

Inventeur(s) : Boudartchouk Jean-Luc

1 La mise en œuvre de la carte archéologique de la France a été à l'origine d'une opération de prospection-inventaire diachronique, limitée à un ensemble géographique choisi pour l'intérêt et la diversité des informations lacunaires qui étaient jusqu'alors en notre possession.

Dans ce secteur pyrénéen comme d'ailleurs sur la totalité de la chaîne montagneuse, l'accent a, de tout temps, été mis sur la recherche préhistorique au détriment des périodes antiques et médiévales. Mais nous savions déjà, d'après les publications, que l'occupation des grottes ne se limitait pas à des contextes préhistoriques et que des carrières de sarcophages étaient connues depuis 1985, hors de toute intégration à notre fichier régional. Il importait donc de rassembler ces données, vérifier les informations anciennes, en rétablissant un contexte scientifique fiable tant en préhistoire qu'en histoire, afin de replacer l'occupation humaine dans une perspective où tous les aspects d'une recherche diachronique seraient pris en compte. Ainsi, il a été possible de " couvrir » la presque totalité de la commune de Lourdes (zones de plaine et de pentes peu abruptes du mont Béout), et d'étendre la prospection sur les communes avoisinantes pour l'étude des gisements de même nature.

3 Pour la préhistoire, des indices d'occupation magdalénienne ont été retrouvés dans les grottes des Espélugues et du col du même nom (faune froide avec une industrie lithique du Paléolithique supérieur), avec une continuité qui, pour celles du col des Espélugues, se poursuit jusqu'au Néolithique comme grotte sépulcrale (ossements humains et racloir 
denticulé). Il en est d'ailleurs de même pour la grotte de la « Tute du Chêne » (herminette polie en silex).

Durant la protohistoire ancienne et récente, des découvertes ponctuelles confirment des permanences d'occupation jusqu'au Bronze ancien et moyen dans la grotte de Milhas 2, alors que les Âges du fer sont majoritairement présents sur le mont Saint-Georges d'Aspin. Il s'agit d'une colline aux flancs abrupts, terminée par deux plateaux morainiques qui ceinturent un pointement rocheux autour du quel ont été retrouvées de nombreuses céramiques attribuables au Premier Âge du fer (urnes globulaires ornées de rainures ou de cordons digités), mais également au Deuxième Âge du fer (céramiques communes indigènes, amphores vinaires italiques Dr. $1 \mathrm{~A}$, céramiques italiques à vernis noir, campanienne A). Ce gisement, jusqu'alors ignoré, s'inscrit dans le groupe (déjà nombreux) d'habitats protohistoriques de hauteur et offre - ceci est particulièrement rare pour notre région - une occupation continue et sans doute stratifiée durant l'ensemble des Âges du fer. Enfin, deux gisements, la grotte du Loup et le rocher du château, ont livré des vestiges céramiques du I ${ }^{\mathrm{er}} \mathrm{s}$. avant J.-C.

5 À l'époque gallo-romaine se rattache l'établissement rural de Lanne d'Anclades (tegulae, imbrices, amphores et céramiques communes du Haut-Empire), mais surtout celui de la colline Saint-Georges d'Aspin. Ici, la terrasse inférieure nord offre une importante stratigraphie au travers de laquelle se distinguent des éléments de toiture (tegulae, clous de charpente )mais aussi du mobilier domestique (céramiques communes, vases à parois fines, verrerie), attestant la persistance de l'habitat durant le $\mathrm{I}^{\mathrm{er}} \mathrm{s}$. après J.-C. La découverte de plusieurs fragments de colonnes en calcaire ainsi que celle plus hypothétique de la partie inférieure d'un autel votif(?) en marbre laissent supposer l'existence d'un temple rural de sommet, cela restant toutefois à confirmer. À cette période, il convient encore d'associer les occupations correspondant au niveau superficiel de la grotte du Loup, qui indiquent une appropriation durant la fin du $\operatorname{IV}^{\mathrm{e}} \mathrm{s}$. et la première moitié $d u V^{e} s$. après J.-C.: céramiques communes, cruches et surtout céramiques "luisantes " à décor guilloché, bol à marli, sigillée africaine "claire $\mathrm{D}$ " correspondant à des formes Hayes 59 et 61 dont certaines sont décorées de motifs estampés de rectangles hachurés aux bords dentelés.

6 Mais c'est le Haut Moyen Âge qui a été le révélateur d'une activité jusqu'alors presque totalement méconnue. En effet, si les premières traces d'extraction de matériaux servant à la fabrication de sarcophages avaient été mises en évidence, dès 1985, au sommet du mont Béout et près de Ségus, nous ne disposions que de très peu d'informations à ce sujet, excepté une première estimation parue en 1991 qui ne recensait que six exploitations. L'opération de prospection systématique qui a couvert une zone plus étendue a ainsi permis la mise au jour de plusieurs carrières jusqu'alors totalement inédites, ce qui suggère un complexe d'extraction privilégiant la production de sarcophages sans équivalent dans le Midi de la France. Le cadre géologique correspond à un calcaire à fossiles (subrécifal de l'Aptien) de couleur gris bleu se comparant au marbre pyrénéen et dont la qualité médiocre a conduit les carriers à une exploitation à un seul niveau d'enlèvement. Les «négatifs » encore visibles (cuves et couvercles), mais aussi les cuves brisées qui n'ont pas été détachées de la roche mère ou qui ont été rejetées dans les déblais, permettent d'évaluer les dimensions des blocs extraits (bisomes dans certains cas), de reconnaître et de restituer les formes toujours trapézoïdales, mais aussi de mieux connaître les conditions techniques d'exploitation de ces carrières à ciel ouvert [ (Fig. $\mathrm{n}^{\circ}$ 1 : Ossen. Zone du mont Béout. Négatif des cuves exploitées en litage sur un seul niveau) 
et (Fig. $n^{\circ} 2$ : Ossen. Zone du mont Béout. Négatif des cuves exploitées en litage sur un seul niveau)]. Les principales zones d'extraction se situent dans le Haut-Béout, sur les communes de Lourdes et d'Ossen, mais aussi à Ségus et Aspin-en-Lavedan. Ainsi, ce sont plus d'une trentaine de zones d'extraction qui ont pu être localisées. L'évaluation quantitative toujours en cours situe la production autour de cinq cents unités, le nombre réel étant vraisemblablement supérieur.

Une première approche de l'aire de répartition de ces sarcophages situe pour l'instant les principaux points de diffusion dans un rayon de $20 \mathrm{~km}$. Sur le plan chronologique, la mise en évidence de plusieurs critères (cuves subrectangulaires à renforts, présence d'une plaque-boucle du $\mathrm{VII}^{\mathrm{e}} \mathrm{s}$., cuve à aménagement céphalique) pourrait provisoirement situer la durée d'exploitation de ces carrières entre le $\mathrm{VI}^{\mathrm{e}} \mathrm{s}$. et le $\mathrm{IX}^{\mathrm{e}} \mathrm{s}$.

8 Au Moyen Âge, hormis quelques céramiques (oules et pégaus) recueillies sur le flanc du rocher qui sert d'assise au château de Lourdes et à proximité de la carrière mérovingienne du Soum de Lanne, il reste encore à signaler deux gisements majeurs déjà connus, mais mal évalués.

9 Le site défensif du Castéra de Julos (565 m d'altitude) établi sur la plate-forme sommitale d'un éperon rocheux comporte des aménagements complexes (fossés, talus, terrasses, motte) de forte ampleur interprétés au XIX ${ }^{e} s$. comme un camp romain (?) alors qu'il s'agit tout simplement d'un site castral médiéval. Un sondage entrepris au niveau de la terrasse aménagée à mi-pente a permis de mettre en évidence trois unités stratigraphiques au travers desquelles le matériel céramique recueilli (oules, cruches à bec tubulaire) suggère une chronologie (encore imprécise) qui ne paraît pas aller au-delà $\mathrm{du} \mathrm{XII}$ s. Le mont Saint-Georges d'Aspin continue a être occupé durant cette période, mais avec des réductions sensibles des zones d'habitats qui semblent se limiter aux alentours de la butte rocheuse aménagée en terrasse semi-circulaire, avec glacis sur le flanc nord. Ce site, mentionné comme défensif au XIII ${ }^{\mathrm{e}}$ s., a livré un matériel céramique très abondant (oules, vases pansus, cruches, vases à liquide, etc.), compris, d'après leur typochronologie, entre le XIII ${ }^{e}$ s. et le XV $\mathrm{XV}^{\mathrm{e}}$. Plus tardivement, une chapelle votive (?) a été construite sur le point culminant et abandonnée antérieurement à la fin du XVIII ${ }^{\mathrm{e}} \mathrm{s}$. (absence de mention sur la carte de Cassini et sur le cadastre napoléonien).

Les résultats scientifiques acquis lors de cette opération sont considérables, notamment sur l'ensemble des carrières à sarcophages mérovingiens. L'importance est telle qu'il conviendra, dans les années à venir, d'étendre cette recherche (au-delà de simples sondages) à l'étude des matériaux et à leur condition d'extraction. De même, l'émergence d'un site comme celui du mont Saint-Georges d'Aspin où l'occupation humaine est assurée depuis la protohistoire jusqu'au Bas Moyen Âge devrait conduire à une réflexion approfondie sur les origines, l'évolution et la fonction d'un site de hauteur jusqu'à son abandon à l'époque moderne.

\section{ANNEXES}


Fig. $\mathrm{n}^{\circ} 1$ : Ossen. Zone du mont Béout. Négatif des cuves exploitées en litage sur un seul niveau

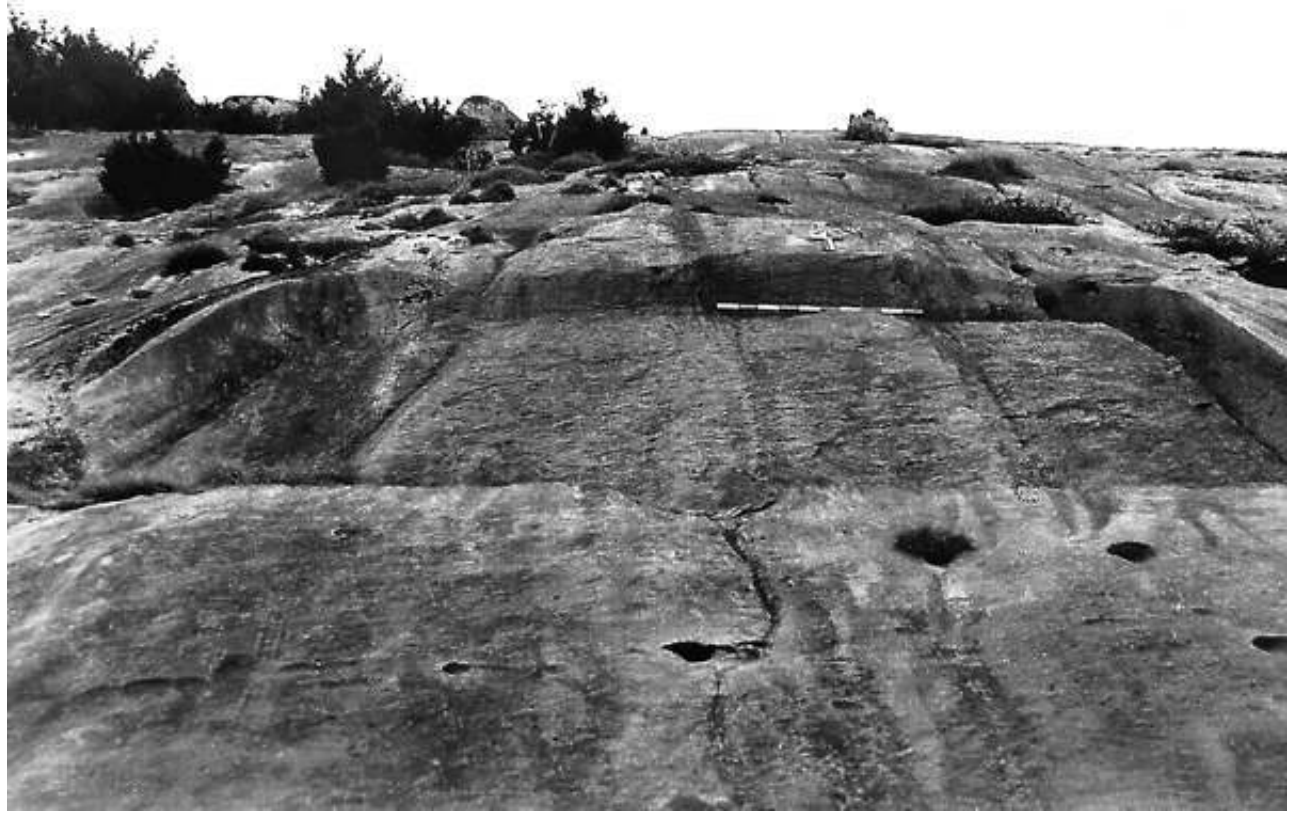

Auteur(s) : Boudartchouk, Jean-Luc. Crédits : Gl, 1997 - CNRS editions, 1998 (1997)

Fig. $n^{\circ} 2$ : Ossen. Zone du mont Béout. Négatif des cuves exploitées en litage sur un seul niveau

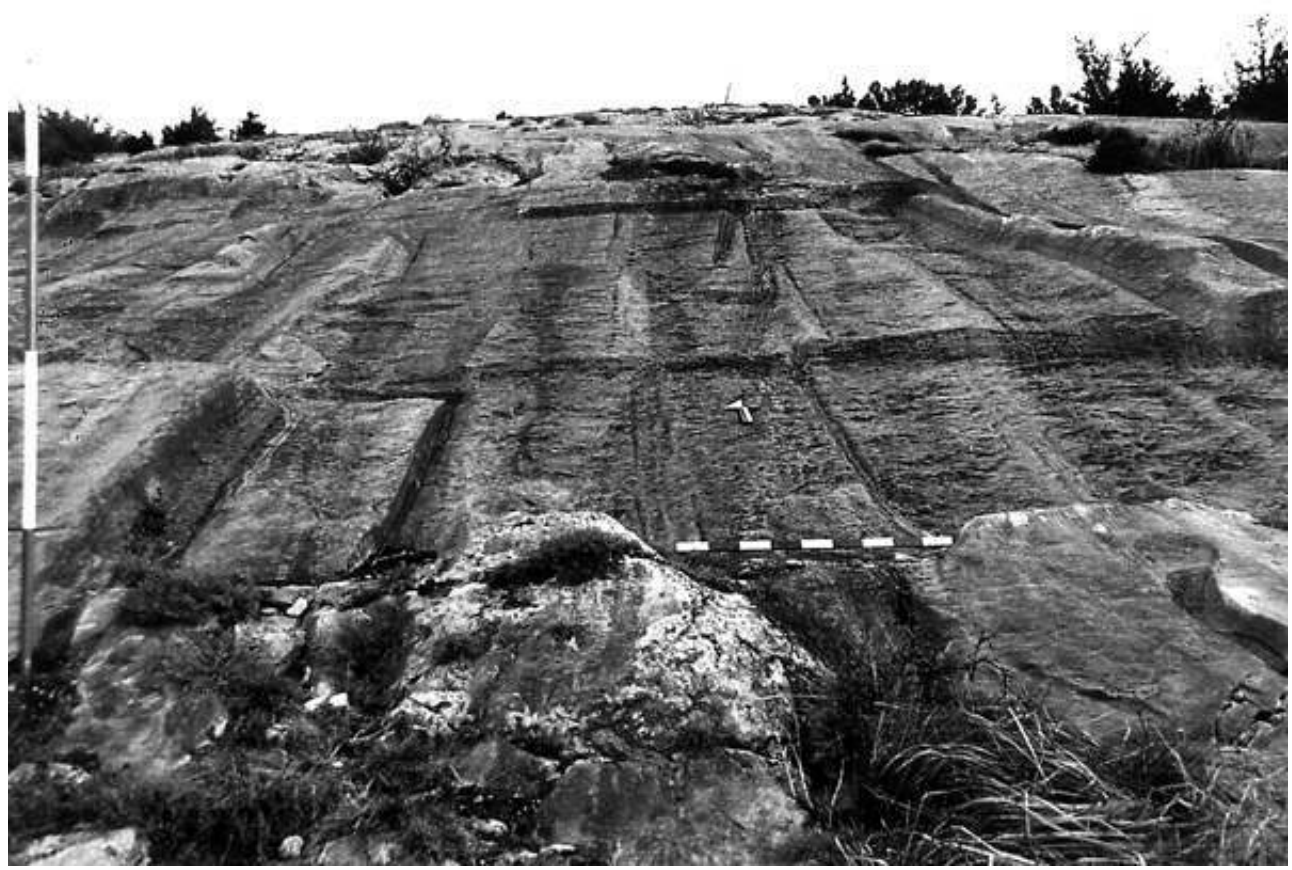

Auteur(s) : Boudartchouk, Jean-Luc. Crédits : GI, 1997 - CNRS Editions, 1998 (1997) 
INDEX

peuple Magdalénien, Mérovingien

operation Prospection (PR)

Index géographique : Midi-Pyrénées, Hautes-Pyrénées (65), Aspin-en-Lavedan, Lourdes, Ossen, Ségus

Index chronologique : bas Moyen Âge, Bronze moyen, Moyen Âge, Néolithique, Premier âge du Fer, âge du Bronze 